\title{
La Administración del Talento Humano en las Pymes en el Ecuador
}

Arteaga Mendoza Erema Liliana Instituto Superior Tecnológico Tsáchilas arteagaliliana@hotmail.com Santo Domingo - Ecuador

Herrera González Clara Pamela Instituto Superior Tecnológico Tsáchilas clarapamela1988@hotmail.com Santo Domingo - Ecuador

Ángel Wilson Villareal Cobeña Instituto Superior Tecnológico Tsáchilas https://orcid.org/0000-0003-0357-0538 wangelvc@hotmail.com angelvillarreal@tsachila.edu.ec Santo Domingo - Ecuador

Patricio Javier López Pérez. Docente de la Universidad Técnica Luis Vargas Torres Sede La Concordia pato92_sto@hotmail.com Quinindé - Ecuador

\section{RESUMEN}

Esta exploración se fundamentada en consolidar de forma general la información referente a la gestión del talento humano en el presente ámbito empresarial, ya que, en las empresas pequeñas y medianas se requieren cambios internos y actualizaciones en sus procesos de gestión direccionados al talento humano, con el objetivo de hallar el avance empresarial recurrente y la máxima potencialización de las capacidades y competencias de su personal. El presente objeto de estudio radica en investigar la gestión del talento humano en funcionalidad a los procesos aplicados por las PYMES. La metodológica aplicado fue el exploratorio, debido al trato inicial de la variable de estudio en el contexto especial investigado, con un enfoque mixto por la utilización de instrumentos de recolección de datos mediante una entrevista. Es de esta forma como se determinó que, en las empresas pequeñas y medianas no se efectúan como corresponde los procesos que intervienen en una eficaz gestión del talento humano, por lo cual se sugiere utilizar tácticas de optimización enfocadas en los elementos bases del desarrollo.

Palabras Claves: Recursos humanos, organización, Comportamiento económico, gestión 


\title{
The administration of human talent in pymes in Ecuador
}

\begin{abstract}
This exploration is based on consolidating in a general way the information regarding the management of human talent in the present business field, since, in small and mediumsized companies, internal changes and updates in their management processes aimed at human talent are required, with the objective of finding recurring business progress and the maximum potentialization of the capacities and competencies of its personnel. The present object of study lies in investigating the management of human talent in functionality to the processes applied by SMEs. The methodological applied was exploratory, due to the initial treatment of the study variable in the special context investigated, with a mixed approach due to the use of data collection instruments through an interview. This is how it was determined that, in small and medium-sized companies, the processes that intervene in an effective management of human talent are not carried out properly, for which it is suggested to use optimization tactics focused on the basic elements of development.
\end{abstract}

Key Words: Human resources, organization, Economic behavior, management

Artículo recibido: 27 marzo 2021 Aceptado para publicación: 30 abril 2021 Correspondencia: arteagaliliana@ hotmail.com Conflictos de Interés: Ninguna que declarar 


\section{INTRODUCCIÓN}

En el Ecuador las empresas tienen la capacidad de crecer y realizarse son aquellas que primordialmente resaltan por la eficaz administración administrativa con la que lideran sus procesos y métodos internos (Agudelo, 2019). En lo relacionado a la gestión del talento humano, en el presente ámbito empresarial el cambio es el exclusivo aspecto que varía todo el tiempo, en donde las empresas se sujetan a la continua utilización de novedosas tecnologías en los procesos productivos y en los enfoques administrativos. Gracias a esto, en las pequeñas y medianas empresas se requieren cambios internos y mejorasen sus procesos de gestión direccionados al talento humano, con el objetivo de hallar el avance empresarial recurrente y la máxima potencialización de las capacidades y competencias de capital humano que las compone (González , 2010). Como respuesta a estos desafíos, muchas empresas eligen por agrandar o predeterminado, direccionarse a novedosas perspectivas que involucren las dimensiones de la gestión del talento humano, como lo son; reclutamiento, selección, inducción, capacitación y evaluación del desarrollo (Montoya \& Boyero, 2016).

En la actualidad las empresas modernas, pequeñas y medianas, en todo el mundo hacen uso de utilidades y prácticas de gestión del talento humano semejantes a las observadas en enormes compañías de renombre mundial, pero este aspecto escasamente es situado en una posición estratégica dentro de una compañía (Polanco, 2013). Uno de los más importantes fundamentos o inconvenientes por lo cual no se proporciona ese gran salto, es la limitada percepción administrativa de parte de los dirigentes de estas pequeñas y medianas empresas, para encaminar eficientemente los procesos que intervienen en la gestión del talento humano, en pro del avance y la optimización del desempeño de la compañía (Medina \& Avila , 2002). Además, a eso sumada la fuerza laboral numerosa, pero carente de talento calificado repercute sobre la construcción de valor de parte de la empresa (Torres \& Jaramillo, 2014).

Sin embargo, a nivel de Ecuador, actualmente en las empresas están empleados con expectativas diferentes sobre el trabajo que tienen que llevar a cabo. Algunos tienen la intención de ayudar con la dirección desde su puesto de trabajo y a otros les atrae la participación ganancial de la empresa (Agudelo, 2019). En otras ocasiones están personas con escasa formación laboral en donde es requisito capacitar antes de tomar su puesto de trabajo, es por esto que, no se transporta a cabo una precisa gestión del talento humano que direccione a los empleados a una eficiente interrelación organizacional (Sandoval, 
2004). Más allá de que las Empresas pequeñas y medianas son sólidas en relación a la naturaleza de sus gestiones y se encargan de la conservación de los usuarios, se ha evidenciado que hay deficiencias en todo el desarrollo de gestión del talento humano, ya que carecen de un direccionamiento estratégico compuesto en todas sus etapas, y más allá de estar en un ámbito de recurrente cambios, estas entidades económicas manejan procesos de administración habituales, sin perspectivas diferenciadoras (Farias , 2014).

En base al tema creado en la presente exploración, se consolida la información en el avance del mismo, donde se describen cada etapa de la exploración, mismo que tiene dentro toda la información medular del presente tema (Contreras \& Castro , 2013). En primera instancia, se denota lo expuesto por distintos autores en referencia a la variable central como lo es la gestión del talento humano, de igual modo, se describen las dimensiones antes ciertas en la matriz de operacionalización de la variable, mismas que aceptan afianzar las conceptualizaciones de la exploración generalmente (Torres \& Jaramillo, 2014). A todo lo mencionado, se denota el apartado donde se establece la metodología usada para el efectivo avance del presente trabajo de exploración, donde está definido la llegada, tipo y enfoque de la exploración, además, de detallar los procedimientos aplicados, y la las técnicas e instrumentos de recolección de datos empleados. Para al final consolidar los datos e interpretación de los resultados que se consiguieron por medio de la aplicación de los instrumentos de recolección de datos antes elaborados (Castro, Luna, \& Erazo, 2020).

\section{ESTRATEGIAS Y MÉTODO Y MATERIALES}

En la presente exploración se tuvo en cuenta como objeto de estudio la Gestión del talento humano en las empresas pequeñas y medianas, es de esta forma, como el avance del tema ya antes citado se sustentó bajo una base de interfaz exploratorio, ya que, en este estudio se intentó una navegación inicial de la variable de estudio, en donde se consiguió la información de fuentes documentales (Hérnandez Sampieri \& Mendoza Torres , 2018). De igual modo, es de enfoque mixto por la utilización de instrumentos de recolección de información, así como son la encuesta y la entrevista. De esta forma se establece que; los estudios exploratorios se hacen cuando el propósito es investigar un tema o inconveniente de exploración poco estudiado, del cual se tienen muchas inquietudes o no se ha abordado antes, cuando la revisión de la literatura mostró que solamente hay guías no investigadas e ideas vagamente similares con el inconveniente de estudio, o bien, si se quiere saber sobre temas y superficies desde novedosas perspectivas (Bernal , 2016). El objetivo por 
el cual se aplicó esta habilidad es comprender lo que un conjunto de individuos piensa y siente sobre un preciso tema. La utilización de la entrevista en la presente exploración se tuvo en cuenta, debido que, es un instrumento subjetivamente eficiente de recolección de datos, debido a que permitió conseguir un panorama inicial del tema de investigado.

\section{ANALISIS DE RESULTADOS}

En este caso, la técnica cualitativa aplicada fue la encuesta, posterior a la realización y ejecución de la entrevista dirigida al personal administrativo y dueños de las PYMES (pequeñas y medianas empresas), se procedió al procesamiento de los datos para la previa análisis de la información obtenida.

Tabla 1. Entrevista a dueños de PYMES

\begin{tabular}{cl}
\hline Preguntas & \multicolumn{1}{c}{ Análisis } \\
\hline & $\begin{array}{l}\text { Esta pregunta permite expresar los siguientes datos: el 10\% de los } \\
\text { países declararon que siempre introducción breve y precisa a los } \\
\text { nuevos empleados con el fin de determinar los objetivos de la } \\
\text { organización, y el 40\% de las personas lo hacen a veces, esta es la } \\
\text { frecuencia con la que realiza este tipo de orientación para los }\end{array}$ \\
$\begin{array}{c}\text { Antes de que el nuevo } \\
\text { personal comience a } \\
\text { trabajar, ¿El tipo de }\end{array}$ & $\begin{array}{l}\text { futuros empleados. Empresas, es porque piensan que los empleados } \\
\text { orientación para alinearse } \\
\text { con los objetivos de la } \\
\text { organización? }\end{array}$ \\
$\begin{array}{l}\text { solo estén relacionados con sus propias funciones, el } \\
\text { funcionamiento de la empresa y finalmente, en cuanto a las } \\
\text { cuestiones planteadas, se determina que casi el 50\% siempre se }\end{array}$ \\
& implementan posicionamiento inicial de nuevos empleados. \\
\hline
\end{tabular}

Respecto a los datos recopilados a través de esta aplicación Para el personal administrativo del país y los propietarios de pequeñas y medianas empresas, el $60 \%$ de la capacitación inicial de inducción

¿Qué tan suficiente es el resumen inicial del nuevo producto personal? que brindan al nuevo personal es modesta porque no han implementado el debido proceso. Por otro lado, el 30\% la gente piensa que esto no es muy suficiente, porque la mayoría de las veces esta acción no se advierte y no se trata como debe ser, en un $10 \%$ de los casos enfatizan en menor medida que están totalmente de acuerdo con la recomendación al nuevo empleado. Pero generalmente señalan que la dotación de personal es un área que es difícil de priorizar.

Antes del análisis de puestos, como aspecto básico de las necesidades de análisis de puestos. Reclutamiento y selección de nuevo personal; el $20 \%$ de las personas mencionó que los aspectos mencionados son ejecutar de manera completamente adecuada; el $30 \%$ de los encuestados dijo que Su nivel de ejecución es moderado, y el $50 \%$ de las personas dice que rara vez Suficiente, porque en la mayoría de los casos, cuando se necesita personal específicamente, los mismos elementos generalmente se seleccionan en función del juicio personal, pero este no es el caso. Análisis adecuado de habilidades, habilidades, experiencia y características. Recursos humanos que son realmente necesarios para realizar el trabajo personal.

Como se muestra los datos correspondientes al plan de previsión. La futura dotación de personal de las pequeñas y medianas 
personal futuro de su empresa?

empresas. Entre ellos, el 10\% de los encuestados dijo que a veces solo estiman el futuro Individuos, depende de su comprensión del período de tiempo requerido Mano de obra adicional, pero no garantiza su durabilidad. Por otro lado, $30 \%$ y $60 \%$ respectivamente que casi nunca harán eso por esta idea. Continúan manteniendo una actitud hacia la administración, lo que demuestra que ciertos la brecha en este enfoque.

Los datos correspondientes a la formación interna Recepción de

¿Está de acuerdo en que la formación interna es Brindar asistencia a los empleados para que desarrollen y mejoren sus capacidades, y capacidad?

\section{¿La empresa utiliza} herramientas estructuradas para evaluar el desempeño de los empleados?

¿Cómo cree que es eficiente la gestión del talento de la empresa?

¿Realizas el proceso de selección del personal?

Antes de seleccionar personal, ¿realiza un análisis de puestos? empleados de pequeñas y medianas empresas con el fin de determinar los criterios para determinar si su implementación permite la mejora, y desarrollo de habilidades y destrezas del talento, de las cuales 60\% Dijo que está de acuerdo con la declaración anterior porque en para los empleados, el individuo es el aspecto más importante y mutuamente beneficioso. En cuanto a la empresa para la que trabaja; seguido por un 60\% Piensan que están totalmente de acuerdo.

Corresponde a herramienta estructurada de evaluación del desempeño de los empleados empresas medianas, de las cuales solo el 20\% dijo la existencia de tales herramientas se utiliza para evaluar eficazmente su desempeño. Por otro lado, en un caso, el $80 \%$ de los colaboradores dijo no utilizar una herramienta tan estructurada no solo puede medir el desempeño de los empleados, sino también, mencionaron que cuando se necesita algún tipo de medición de actividad ejecutado por empleados, que se basa en completa las actividades asignadas.

Como última parte del cuestionario, las siguientes preguntas están relacionadas con después de responder las preguntas anteriores y analizar rápidamente preguntas el proceso de gestión del talento involucrado Nota: Entre las respuestas recibidas, el $40 \%$ de las personas piensa que la gestión del talento el personal que opera el sector económico es moderadamente eficiente; igual a en el mismo porcentaje, dijeron que la ejecución de su proceso es regular, por lo que finalmente, el porcentaje es menor, pero la correlación es la misma que antes al 20\% Requieren muy poca gestión de recursos humanos Eficiente

$\mathrm{Si}$, el proceso de reclutamiento efectuado en la pyme se ejecuta de manera tradicional,

llámese a esto el hecho de no utilizar técnicas de reclutamiento como tal, que garanticen la captación de personal idóneo, sino más bien se manejan por medios de consumo local para publicar la solicitante de personal, como son las radiodifusoras. Al generarse una vacante ya sea por despido o por abandono voluntario de un puesto de trabajo, es casi inmediato el llamamiento de posibles candidatos para el cargo, cabe recalcar que dependiendo cual sea el cargo vacante, es la formalidad que le dan al proceso.

Se desconoce la terminología como tal, sin embargo, limitadamente este es realizado por el encargado de receptar los currículos de quienes se acerquen al establecimiento o de quienes hacen llegar su información de manera digital (en ocasiones es el dueño quien se encarga del proceso). En esta parte se menciona que de manera general se identifican las características que debe tener el nuevo trabajador, mas no se analiza y deja por sentado cuales son realmente los requerimientos, habilidades, características, entre 


\begin{tabular}{|c|c|}
\hline & $\begin{array}{l}\text { otros aspectos que necesariamente debe tener quien solicita la } \\
\text { vacante. }\end{array}$ \\
\hline $\begin{array}{l}\text { ¿Ha establecido una } \\
\text { descripción del puesto en } \\
\text { el manual de trabajo o } \\
\text { algún otro documento que } \\
\text { acredite su existencia? }\end{array}$ & $\begin{array}{l}\text { No, la descripción del trabajo sobre si establecieron la empresa, la } \\
\text { respuesta está en la comprensión de funciones, responsabilidades y } \\
\text { dependencias. Cuando nuevos empleados ingresan a la empresa, se } \\
\text { verán reflejados verbalmente a los nuevos empleados. No se trata } \\
\text { de ningún tipo de manual que haya sido previamente definido. }\end{array}$ \\
\hline
\end{tabular}

\begin{tabular}{c}
\hline ¿Cree que es necesario \\
planificar la dotación de \\
personal futuro para las \\
pequeñas y medianas en el \\
Ecuador?
\end{tabular}

A veces es, por ejemplo, dependiendo del alcance comercial de la empresa, empresas que generalmente venden bienes, productos o materias primas, gestionar empleados durante la llamada temporada alta de ventas. Deben tener suficiente talento para cubrir sus afirmar. Pero, en general, este no es un proceso utilizado habitualmente por las pymes.

\begin{tabular}{cl}
\hline ¿Ha realizado formación & Sí, cuando se contrata e integra a nuevos empleados en sus puestos, \\
de inducción para nuevos & obtienen una breve descripción de las expectativas que se esperan \\
empleados? Si su & cumplir igualmente eficaz. Explore la universalidad de las áreas de \\
respuesta es sí, ¿cómo & aplicación, Inferir que la inducción ocurre más a través del acuerdo \\
lleva a cabo la & en lugar de ser considerada es fundamental para el desarrollo \\
capacitación de inducción & efectivo de nuevos colaboradores.
\end{tabular}
para nuevos empleados?

¿Cómo evalúa el desempeño de los empleados de la empresa?

Cabe mencionar que la evaluación del desempeño del personal de la empresa no Puede realizarse bajo cualquier tipo de esquema o estructura, y esta evaluación es se ha implementado, pero el desempeño del socio se mide en función del desempeño del socio. Área de trabajo si es parte del personal responsable de la parte de ventas, Por otro lado, esto se basa en su nivel de ventas mensual, si es parte del personal de la oficina de la empresa, porque solo en este caso, su efectivo o no puede completar las tareas con eficacia en el desarrollo diario.

\begin{tabular}{lll}
\hline ¿Cómo crees que es la & Proporcione un punto de vista general y céntrese en gestión de \\
gestión del talento? & recursos humanos. Creo que este proceso no está en el conjunto \\
¿Pequeñas y medianas & ejecutado correctamente por el responsable de la PYME es \\
empresas en el Ecuador? & $\begin{array}{l}\text { evidentemente, no todos los procesos tratados en este proceso } \\
\text { momento. A medida que continúe la gestión o ejecución de dichos } \\
\text { aspectos Según la experiencia, por supuesto, no para todos, sino } \\
\text { para la gran mayoría de PYMES estándares. }\end{array}$ \\
\hline
\end{tabular}

Nota: Se describe los resultados realizados a los dueños de la PYMES, en este caso se tomo para el análisis varios locales activos que han sobrevivido a esta pandemia y que piensas salir a delante mejorando su perspectiva empresarial.

\section{Elaborado por: Grupo investigador}

\section{DISCUSIÓN}

Resultados obtenidos en base a la aplicación de la herramienta de recolección en los datos mencionados anteriormente en este trabajo, es posible determinar el objetivo general del estudio de referencia, es decir, la gestión del talento (Contreras \& Castro , 2013). Las pequeñas y medianas empresas en el Ecuador tienen una eficiencia media; esto encontrado en la última pregunta de la herramienta de recopilación de datos, en este caso 
la parte decisiva de la encuesta tras responder las preguntas anteriores y analizó rápidamente el proceso involucrado gestión del talento considerada en la respuesta entre los entrevistados obtenidos, el $40 \%$ cree que la gestión del talento que realizan estas personas la eficiencia de las unidades económicas es media, lo que también confirma la última pregunta de la entrevista.

En términos generales, esto puede suceder debido a un defecto en el proceso, participar en la gestión de recursos humanos, que es su negocio típico. Casi no hay diferencia entre la naturaleza tradicional y empírica. Corriendo en una de estas empresas, esto está comprobado en los siguientes aspectos: Investigación, entre ellos, en una de las preguntas sobre el análisis de puesto anterior reclutamiento y selección de personal el 50\% de los encuestados dijo que este proceso No muy adecuado, porque en la mayoría de los casos, cuando es necesario personal específico, la elección del mismo personal generalmente se basa en el juicio personal, más no, las habilidades, habilidades, experiencia y características deben analizarse adecuadamente. Personas verdaderamente necesarias para el desarrollo del puesto de trabajo, y Necesidades de personal.

De la misma forma, el 50\% de las personas casi siempre realizan orientación inicial a sus hijos. Personal recién contratado, y luego en otra parte, principalmente indican el $60 \%$ de la formación inicial de inducción que proporcionan a los nuevos empleados es moderada. Adecuado, esto se debe a que no realizaron el debido proceso, porque esta acción es en la mayoría de los casos, no llamará la atención y no se manejará adecuadamente. Esto es lo que yo se determinar que las PYMES, en el Ecuador no se encuentran implementadas en su totalidad Intervenir en el proceso de gestión eficaz de talentos. Toda Esta información es completamente relevante para la base de futuras investigaciones.

\section{CONCLUSIÓN}

En la actualidad, la gestión del talento se puede ver desde los siguientes aspectos diferentes visiones que satisfacen las necesidades de cada organización, su enfoque situacional depende del medio ambiente, la tecnología utilizada políticas internas, internas, lineamientos actuales, ideas la autoridad administrativa gestionada por la organización, lo más importante, su número y dado diferentes diferencias entre diferentes conceptos. Personas y organización. Según los resultados obtenidos a través de la tecnología, Las herramientas de encuesta utilizadas en la encuesta, posiblemente Indica que hay defectos en el proceso involucrado gestión del talento realizada por pequeñas y medianas empresas en el Ecuador para hacerlo moderadamente ejecutado eficiente, 
gracias en gran parte a la experiencia y pocos conceptos formales Los procesos internos de estas unidades económicas.

Además, se extrae de la investigación es que el ejercicio del procedimiento el talento gerencial adecuado puede hacer que constituya tales como: reclutamiento, selección, inducción, capacitación y evaluación Personal, además de análisis y descripciones de puestos, Apropiadamente, porque lo más importante es formar parte del capital humano de las pequeñas y medianas empresas en el Ecuador lo mejor para cubrir puestos vacantes y generar altos ingresos nivel de productividad.

\section{REFERENCIAS BIBLIOGRÁFICAS}

Agudelo, B. (2019). Formación del talento humano y la estrategia organizacional en empresas de Colombia. Revista Entramado, 15(116-137). Obtenido de http://www.scielo.org.co/pdf/entra/v15n1/1900-3803-entra-15-01-116.pdf

Bernal , C. (2016). Metodologia de la investigación (Cuarta ed.). Colombia: Prentice Hall. Castro, K., Luna, K., \& Erazo, J. (2020). GESTIÓN DE TALENTO HUMANO PARA LA MEJORA LABORAL EN EL BANCO SOLIDARIO. Revista de Estudios Interdisciplinarios en Ciencias Sociales, 22(1), 184 - 203. Obtenido de file://C:/Users/Ramiro\%20Guaman/Downloads/Dialnet-

GestionDeTalentoHumanoParaLaMejoraLaboralEnElBanco-7200012.pdf

Contreras , F., \& Castro , G. (2013). Liderazgo, poder y movilización organizacional. Revista Estudios Gerenciales, 29(126), 72-76. Obtenido de https://www.redalyc.org/pdf/212/21228397008.pdf

Farias , P. (2014). Estrategias de marketing utilizadas por las empresas chilenas para incrementar el valor de los clientes. Cuadernos de Administración, 30(51), 7. Obtenido de http://www.scielo.org.co/pdf/cuadm/v30n51/v30n51a02.pdf

González , L. (2010). Modelos de Dirección de Recursos Humanos. Papeles del Spicológo, $\quad 5(72), \quad 25 \quad$ - $\quad 34 . \quad$ Obtenido de http://cursos.itam.mx/sastre/lecturas/Modelos\%20de\%20direccion\%20de\%20recurs os\%20humanos.PDF

Hérnandez Sampieri, R., \& Mendoza Torres , C. (2018). Metodología de la investigación: las rutas cuantitativa, cualitativa y mixta (Sexta ed.). México: McGraw Hill.

Lockward, A. (2011). El rol de la confianza en las organizaciones a través de los distintos enfoques o pensamientos de la administración. Revista Ciencia y Sociedad, 36(3), 464 - 502. Obtenido de https://www.redalyc.org/pdf/870/87022526005.pdf 
Mariño, J. (2011). DISEÑO DE PUESTOS DE TRABAJO EN UNA ORGANIZACIÓN LOCAL DE GESTIÓN DE LA ACTIVIDAD FÍSICA Y EL DEPORTE. Revista Ciencia en su PC, 1(3), 52 -66. Obtenido de https://www.redalyc.org/pdf/1813/181322267005.pdf

Medina , A., \& Avila , A. (2002). Evolución de la teoria administrativa. una vision desde la psicología organizacional. Revista Cubana de Psicologia, 19(3), 1-11. Obtenido de http://pepsic.bvsalud.org/pdf/rcp/v19n3/12.pdf

Montoya, C., \& Boyero, M. (2016). EL RECURSO HUMANO COMO ELEMENTO FUNDAMENTAL PARA LA GESTIÓN DE CALIDAD Y LA COMPETITIVIDAD ORGANIZACIONAL. Revista Científica "Visión de Futuro", 20(2), 1-20. Obtenido de https://www.redalyc.org/pdf/3579/357947335001.pdf

Pazmay, S. G., \& Ortiz, Á. R. (19 de junio de 2018). Clima organizacional en las industrias ecuatorianas de calzado. Revista Cuadernos Latinoamericanos de Administración, $\quad 14(26), \quad 1-\quad 19 . \quad$ Obtenido de https://www.redalyc.org/jatsRepo/4096/409656163003/html/index.html

Peñaloza, M. (2005). El Mix de Marketing: Una herramienta para servir al cliente. Actualidad Contable Faces, 8(10), 71-81. Obtenido de https://www.redalyc.org/pdf/257/25701007.pdf

Polanco, Y. (2013). El proceso de administración de recursos humanos. Enfoque hacia los expatriados. Análisis Económico, 28(67), 77 - 91. Obtenido de https://www.redalyc.org/pdf/413/41329570006.pdf

Sandoval , M. D. (2004). Concepto y dimensiones del clima organizacional. Revista de Administración, $\quad$ 10(27), 78-82. Obtenido de https://moodle2.unid.edu.mx/dts_cursos_mdl/lic/AE/EA/AM/02/Concepto_dimensi ones.pdf

Torres , J., \& Jaramillo, O. (2014). DISEÑO Y ANÁLISIS DEL PUESTO DE TRABAJO. Barranquilla: X.Pres estudio Gráfico y digital. Obtenido de https://corladancash.com/wp-content/uploads/2020/01/Diseno-y-analisis-delpuesto-de-Jose-Luis-Torres-Laborde.pdf 